Original Paper http://ajol.info/index.php/ijbcs http://indexmedicus.afro.who.int

\title{
Evaluation de l'infestation des Loranthaceae sur les ligneux des agroécosystèmes de la région du Sud-Comoé (Côte d'Ivoire)
}

\author{
Denis-Esdras Anoh AMON ${ }^{1 *}$, Dodiomon $\mathrm{SORO}^{1,2}$ et Dossahoua TRAORE ${ }^{1}$ \\ ${ }^{1}$ Laboratoire de Botanique, U.F.R. Biosciences, Université Félix Houphouët-Boigny, \\ 22 BP 582 Abidjan 22, Côte d'Ivoire. \\ ${ }^{2}$ Centre National de Floristique (CNF), Université Félix Houphouët-Boigny, Côte d'Ivoire. \\ *Auteur correspondant, E-mail: amonson77@yahoo.fr, Tel. : 0022508182805
}

\section{RESUME}

En Côte d'Ivoire, les plantes vasculaires parasites de la famille des Loranthaceae constituent une menace majeure pour de nombreuses essences fruitières et sauvages compte tenu de l'ampleur des dégâts. La présente étude a été entreprise dans l'optique de contribuer à mieux connaître les Loranthaceae et leur parasitisme sur la flore ligneuse des agro-écosystèmes (cacaoyers, caféiers) dans le Sud-Comoé (Côte d'Ivoire). Dans les vergers, les espèces de Loranthaceae rencontrées et les arbres hôtes ont été recensés. Sur chaque taxon, le nombre de touffes a été compté. Dans les agroécosystèmes, 115 espèces hôtes reparties dans 77 genres et 34 familles sont parasitées par 7 espèces de Loranthaceae. Tapinanthus bangwensis est l'espèce ubiquiste la plus abondante de toutes les Loranthaceae rencontrées. Le taux moyen d'infestation des taxons hôtes inventoriés est de 37,94 p.c. (pourcent) et l'intensité moyenne d'infestation est de 8,15 touffes/arbre. Le parasitisme des Loranthaceae est une menace à considérer. Il nous paraît donc essentiel d'élaborer des programmes de recherche de lutte ciblée pour les essences fruitières, source de revenu pour les paysans.

(C) 2015 International Formulae Group. All rights reserved.

Mots clés: Loranthaceae, taxons hôtes, taux d'infestation, intensité d'infestation, lutte ciblée.

\section{Evaluation of the infestation of wood Loranthaceae on agroecosystems of the Sud-Comoé region (Côte d'Ivoire)}

\begin{abstract}
In Côte d'Ivoire, parasites vascular plants of the family Loranthaceae are a major threat to many species and wild fruit given the extent of the damage. The present study was undertaken with the aim to contributing to better understanding Loranthaceae and parasitism on the woody flora of agroecosystems (cocoa, coffee) in the Sud-Comoé (Côte d'Ivoire). In orchards, Loranthaceae species found and host trees have been identified. On each taxon, the number of clumps was counted. In agroecosystems, 115 host species distributed in 77 genera and 34 families are parasitized by 7 species of Loranthaceae. Tapinanthus bangwensis is the most abundant ubiquitous species of all Loranthaceae found. The average rate of infestation of inventoried hosts taxa is 37.94 p.c. (per cent) and the average intensity of infestation is 8.15 tufts/tree. Parasitism of Loranthaceae is a threat to
\end{abstract}


be considered. We therefore consider it essential to develop targeted control of research programs for fruit trees, source of income for farmers.

(C) 2015 International Formulae Group. All rights reserved.

Keywords: Loranthaceae, host taxa, infestation rates, infestation intensity, targeted control.

\section{INTRODUCTION}

Les Loranthaceae sont des arbrisseaux, chlorophylliens qui vivent en hémiparasites sur les branches d'autres arbres cultivés et spontanés (Amon, 2014). Leur implantation sur les branches d'un sujet provoque une diminution de la croissance de l'individu hôte. Selon Boussim (2002), les plantes parasites, une fois fixées sur la branche hôte, établissent des liaisons fonctionnelles avec l'appareil conducteur de l'hôte grâce à un suçoir. Elles prélèvent ainsi l'eau, les sels minéraux nécessaires dont elles ont besoin pour leur développent sur les hôtes. Les plantes parasites représentent donc un véritable danger pour de nombreuses espèces ligneuses cultivées et sauvages des zones tempérées et tropicales. Leur mode de vie parasitaire provoque des pertes de rendement souvent considérables des sujets parasités (Salle, 2004; Boussim et al., 2012). Le parasitisme de ces hémiparasites est un problème écologique et agronomique. Les hémiparasites sont largement distribués dans les régions tropicales. Ils appartiennent à l'ordre des Santalales et à la famille des Loranthaceae qui compte 77 genres et plus de 950 espèces (Dibong et al., 2009) en Afrique, notamment en Côte d'Ivoire, où il existe, selon les travaux de Aké-Assi (2001) et Amon (2014), 24 espèces. Depuis quelques décennies, suite aux divers changements climatiques de la sous-région, conséquence de la variation écologique liée à la destruction des formations végétales primaires, les hémiparasites sont devenus une menace réelle pour les arbres et arbustes cultivés (avocatier, goyaviers, colatiers, agrumes...) et sauvages contre laquelle une lutte énergique s'avère indispensable compte tenu de l'ampleur des dégâts. La présente étude a été entreprise dans l'optique de contribuer à mieux connaître les Loranthaceae et leur parasitisme sur la flore ligneuse dans les agroécosystèmes. Il s'agit de: (1) inventorier les espèces parasites en cause, (2) évaluer le degré de parasitisme des Loranthaceae sur les essences ligneuses des agroécosystèmes.

\section{MATERIEL ET METHODES \\ Site de l'étude}

Le site d'étude choisi est le Sud-Comoé (Figure 1), une région administrative qui couvre une superficie de $800 \mathrm{~km}^{2}$. Il regroupe trois départements (Aboisso, Grand-Bassam, Adiaké). Le département d'Aboisso, de coordonnées géographiques $5^{\circ} 66^{\prime}$ et $5^{\circ} 28^{\prime}$ de latitude Nord et de $3^{\circ} 12^{\prime}$ et $-3^{\circ} 20^{\prime}$ de longitude Ouest, est situé à $116 \mathrm{~km}$ d'Abidjan (Koua, 2007). Quant au département d'Adiaké $\left(5^{\circ} 28^{\prime}\right.$ et $5^{\circ} 21^{\prime}$ de latitude Nord et de $3^{\circ} 16^{\prime}$ et $-3^{\circ} 08^{\prime}$ de longitude Ouest), est distant de $94 \mathrm{~km}$ d'Abidjan (Anader, 2003b). Concernant le département de Grand-Bassam, compris entre $5^{\circ} 26^{\prime}$ et $5^{\circ} 13^{\prime}$ de latitude Nord et de $3^{\circ} 44^{\prime}$ et $-3^{\circ} 58^{\prime}$ de longitude Ouest, est situé à 43 km d'Abidjan (Anader, 2003a).

Le choix de cette région est motivé par une expansion croissante des Loranthaceae sur les arbres et arbustes des agroécosystèmes qui affectent la croissance et le potentiel de production des essences fruitières. Le climat de la zone d'étude est de type sub-équatorial de transition, communément appelé climat attiéen. Les moyennes pluviométriques annuelles est de $1500 \mathrm{~mm}$. La température moyenne annuelle est de $27{ }^{\circ} \mathrm{C}$, varie mensuellement entre 25 et $26{ }^{\circ} \mathrm{C}$. La 
végétation est caractérisée par des ilots forestiers, des forêts classées et des formations anthropiques (champs, plantations).

\section{Matériel}

Le matériel végétal a été composé de la flore ligneuse des agroécosystèmes et des espèces de Loranthaceae. Le matériel technique comprend un appareil de positionnement géographique (GPS), un appareil photo numérique, une paire de jumelles, des cordes et un ruban-mètre.

\section{Méthodes}

Les travaux se sont déroulés de 2007 à 2009. Des investigations ont été menées dans les agroécosystèmes de la région du SudComoé, notamment dans les exploitations cacaoyères et caféières. Les exploitations ont été choisies par un tirage au sort, de façon aléatoire, sur la base de la liste de l'ensemble des plantations de cacaoyers et de caféiers répertoriées par la Direction Départementale de l'Agriculture.

La méthode de relevés de surface a été utilisée pour collecter les données sur le terrain (Hall et Swaine, 1981). Elle a consisté à délimiter des parcelles dans les plantations. Des placettes d'un hectare de superficie chacune $(100 \mathrm{~m} \quad \mathrm{x} \quad 100 \mathrm{~m})$, disposées alternativement à différents endroits dans les plantations sont retenues pour cet inventaire de la flore ligneuse. Chaque individu est caractérisé par son nom.

Pour un inventaire floristique minutieux, la parcelle a été subdivisée en des bandes de 10 mètres de largeur et de 100 mètres de longueur. Par bande, les individus parasités et sains, les touffes des parasites sur chaque individu parasité ont été dénombrés. Les données recueillies ont permis de déterminer l'état d'infestation comme suit:

- Taux d'infestation $(T x)=$ (nombre d'individus parasités/nombre total d'individus présents) x100;
- L'intensité d'infestation (Ii) = nombre total de touffes de Loranthaceae/nombre total d'individus infestés recensés.

Les analyses statistiques des résultats ont été faites avec le logiciel Excel 5.0.

\section{RÉSULTATS}

\section{Flore des agroécosystèmes inventoriés}

Le Tableau 1 présente la composition floristique des taxons hôtes recensés dans les agroécosystèmes. On note une forte proportion des espèces fruitières (avocatiers, agrumes...) présentes dans les plantations. Au total, 115 espèces reparties dans 77 genres et 34 familles, parasitées par sept espèces de Loranthaceae ont été inventoriées dans les agroécosystèmes de la zone étudiée. Les sept espèces parasites rencontrées sont Tapinanthus bangwensis (Engl. et K. Krause) Danser (Figure 2), Tapinanthus belvisii (DC) Danser (Figure 3), Tapinanthus sessilifolius var. glaber (P. Beauv.) Van Tiegh. (Figure 4), Phragmanthera capitata (Spreng.) Ballé (Figure 5), Phragmanthera capitata var. alba (Spreng.) Ballé, Globimetula braunii (Engl.) Van Tiegh. et Globimetula dinklagei subsp. assiana (Engl.).

Les données du Tableau 1 montrent que sur 12086 individus inventoriés, 4586 sujets sont parasités (Tableau 1). Les familles des espèces hôtes les plus parasitées sont Caesalpiniaceae (13 taxons, soit 48,23 p.c.), Moraceae (10 taxons, soit 29,41 p.c.), Euphorbiaceae et Mimosaceae avec 9 taxons chacun ( 26,47 p.c.), Apocynaceae et Rutaceae avec 7 taxons chacune, soit 20,59 p.c. De même, 14 familles sont les moins infestées avec une seule espèce chacune. C'est le cas de la famille des Bignionaceae avec Newbouldia laevis, la famille des Cecropiaceae avec Cecropia peltata, la famille des Lauraceae avec Persea americana, la famille des Solanaceae avec Solanum melongena. 


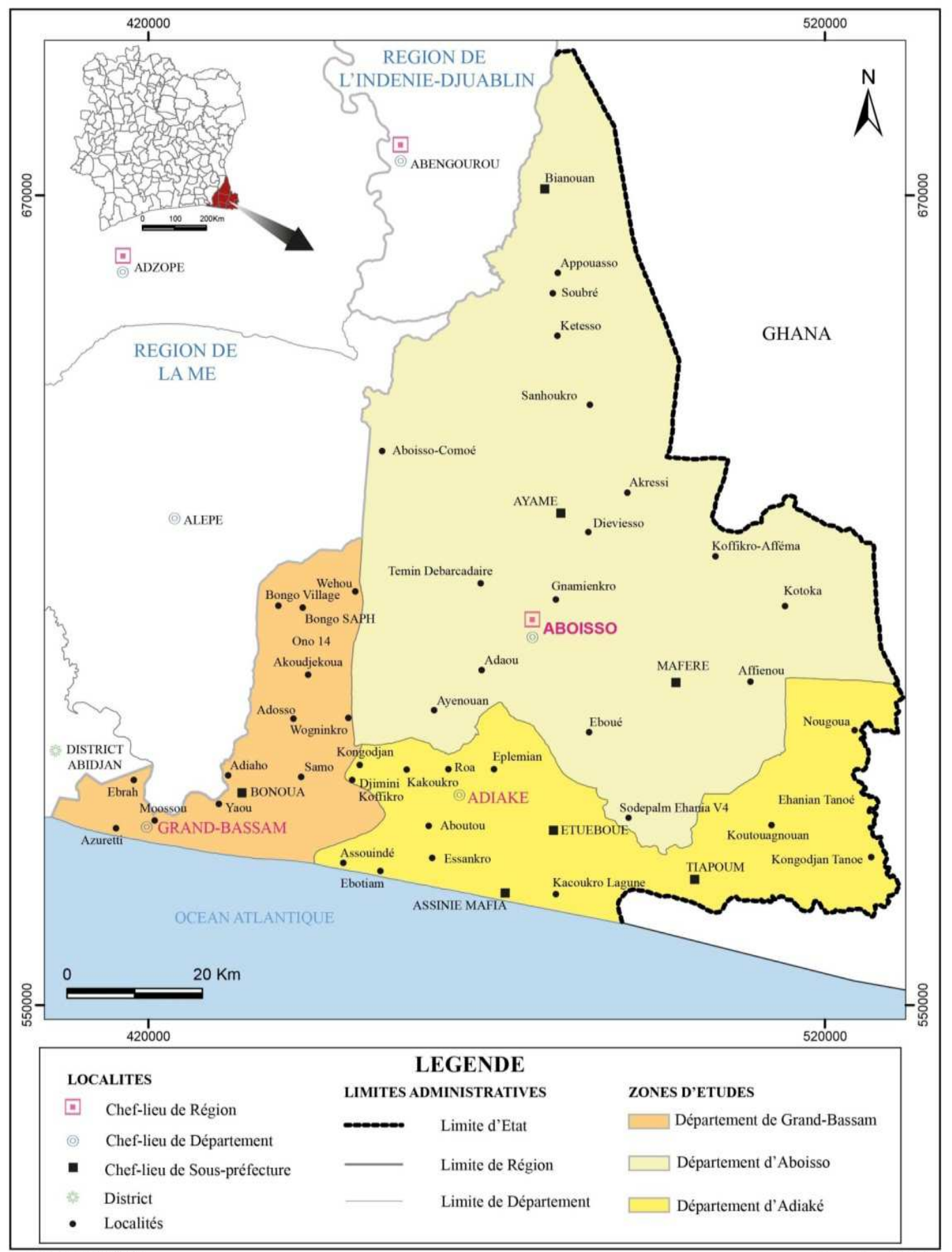

Source : BNETD/CCT

Conception : AMON

Figure 1: Carte administrative de la région du Sud-Comoé (Sud-Est, Côte d'Ivoire). 


\section{Etat d'infestation des arbres et arbustes recensés}

En ce qui concerne le degré d'infestation, le Tableau 1 indique que le taux de parasitisme varie selon les espèces hôtes. Il est compris entre 1,46 p.c. et 84,95 p.c. Les taxons attaqués sont Cecropia peltata $(84,95$ p.c.), Spondias mombin (75,24 p.c.), Acacia mangium (71,42 p.c.), Theobroma cacao (67,27 p.c.), Hevea brasiliensis (58,18 p.c.), Pycnanthus angolensis (56,20 p.c.), Cola nitida (54,98 p.c.), Funtumia elastica $(51,78$ p.c.), Anthocleista djalonensis (50,98 p.c.), Bombax buonopozense (49,80 p.c.), Gmelina arborea (50 p.c.), Rauwolfia vomitoria (44,97 p.c.) et Persea americana (44,34 p.c.). On observe également des pieds de Mangifera indica parasités par les Loranthaceae $(1,88$ p.c.).

Quant à l'intensité d'infestation, on note chez Acacia mangium $(19,60$ touffes/arbre), Hevea brasiliensis $(14,14$ touffes/arbre), Tectonia grandis $(13,28$ touffes/arbre) et Persea americana (12,76 touffes/arbre) contre 1 touffe/arbre chacune chez Psidium pyriferum et Citrus reticulata.

Onze espèces fruitières bien connues, sont diversement parasitées par les Loranthaceae dans les agroécosystèmes. Leur taux d'infestation oscille entre 1,46 et 58,33 p.c. On cite Anacadium occidentale (58,33 p.c.), Cola nitida (54,98p.c.), Persea americana (44,34 p.c.), Citrus grandis $(35,71$ p.c.), Citrus limon (35,41 p.c.), Citrus reticulata (33,33 p.c.), Citrus sinensis (27,31 p.c.). On note aussi une forte sensibilité des sujets des cultures pérennes. Leur taux d'infestation oscille entre 6,36 et 67,27 p.c. Il s'agit de Theobroma cacao (67,27 p.c.), Hevea brasiliensis (58,18 p.c.), Coffea arabusta (10,51 p.c.) et Coffea canephora var. robusta (6,36 p.c.). Theobroma cacao est l'espèce des cultures de rente la plus parasitée (67,27 p.c.).
Globalement, sur 12086 individus inventoriés, 4586 sont parasités. Le taux moyen d'infestation des essences ligneuses des agro-écosystèmes du Sud-Comoé est de 37,94 p.c. et l'intensité d'infestation de 8,15 touffes/arbre (Tableau 1).

Le Tableau 2 relatif au parasitisme des Loranthaceae sur les ligneux présente Tapinanthus bangwensis comme l'espèce la plus fréquente (ubiquiste) et la plus abondante dans la région, avec un nombre de touffes moyen de 39,84 p.c. Elle est suivie dans l'ordre décroissant par Phragmanthera capitata (24,7 p.c.), Phragmanthera capitata var. alba (17,61 p.c.), Tapinanthus sessilifolius var. glaber (10,51 p.c.), Globimetula braunii (6,01 p.c.), Tapinanthus belvisii (0,21 p.c.) et Globimetula dinklagei (0,48 p.c.). Par ailleurs, Tapinanthus belvisii est la seule espèce rencontrée dans la partie littorale de la région d'étude (Grand-Bassam).

\section{DISCUSSION}

\section{Espèces de Loranthaceae inventoriées}

Sept espèces de Loranthaceae ont été inventoriées et identifiées sur les 11 recensées dans la zone forestière ivoirienne. Cette différence de nombre d'espèces est due à l'étendue de la zone d'étude et au milieu écologique étudié. Par contre, ce nombre est supérieur à celui de 5 espèces inventoriées par Soro (2010) dans les vergers à l'Ouest du pays. Tapinanthus bangwensis et Phragmanthera capitata sont des espèces ubiquistes, les plus abondantes des 7 espèces de Loranthaceae inventoriées avec une prédominance de Tapinanthus bangwensis. Le fort taux de parasitisme de ce parasite à déjà été observé par Soro et al. (2004) sur les Karités du parc naturel de Tengréla. Tapinanthus belvisii est la seule espèce rencontrée dans la partie littorale de la zone d'étude. Des résultats similaires ont été obtenus par Amon (2006) dans le département de Grand-Bassam sur les ligneux. 


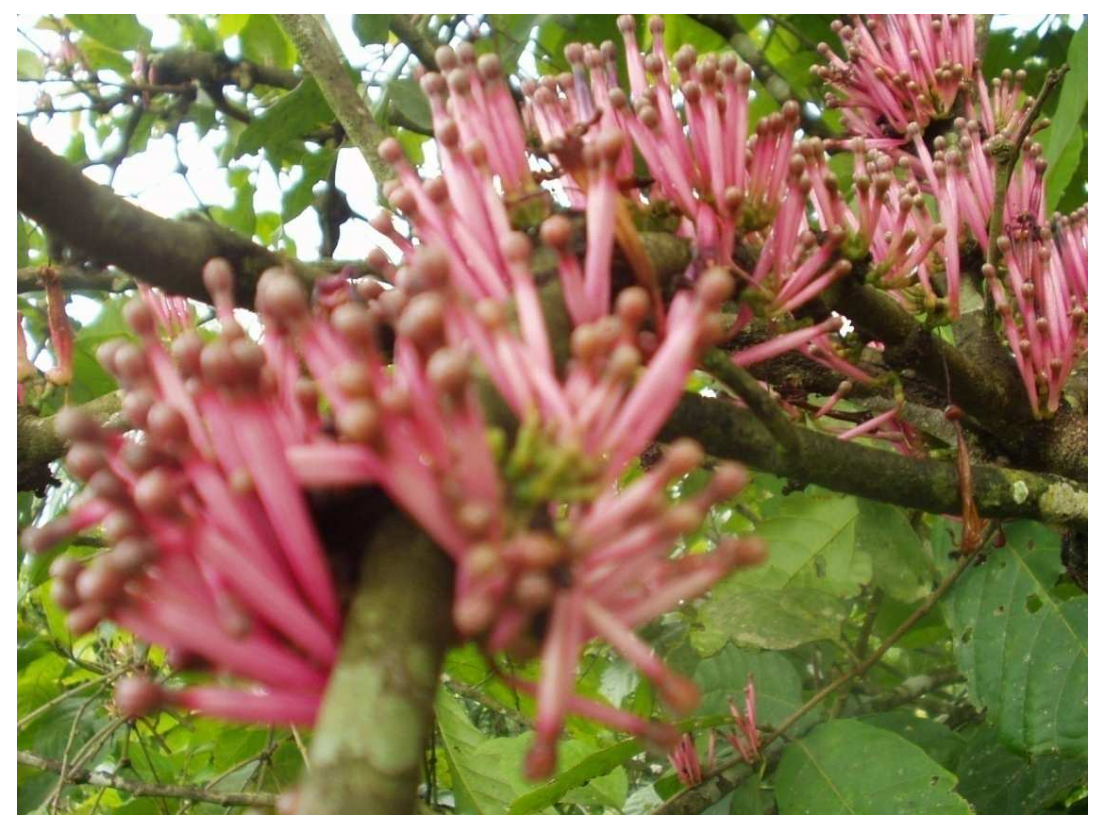

Figure 2: Rameaux florifères de Tapinanthus bangwensis (Engl. et K. Krause) Danser.

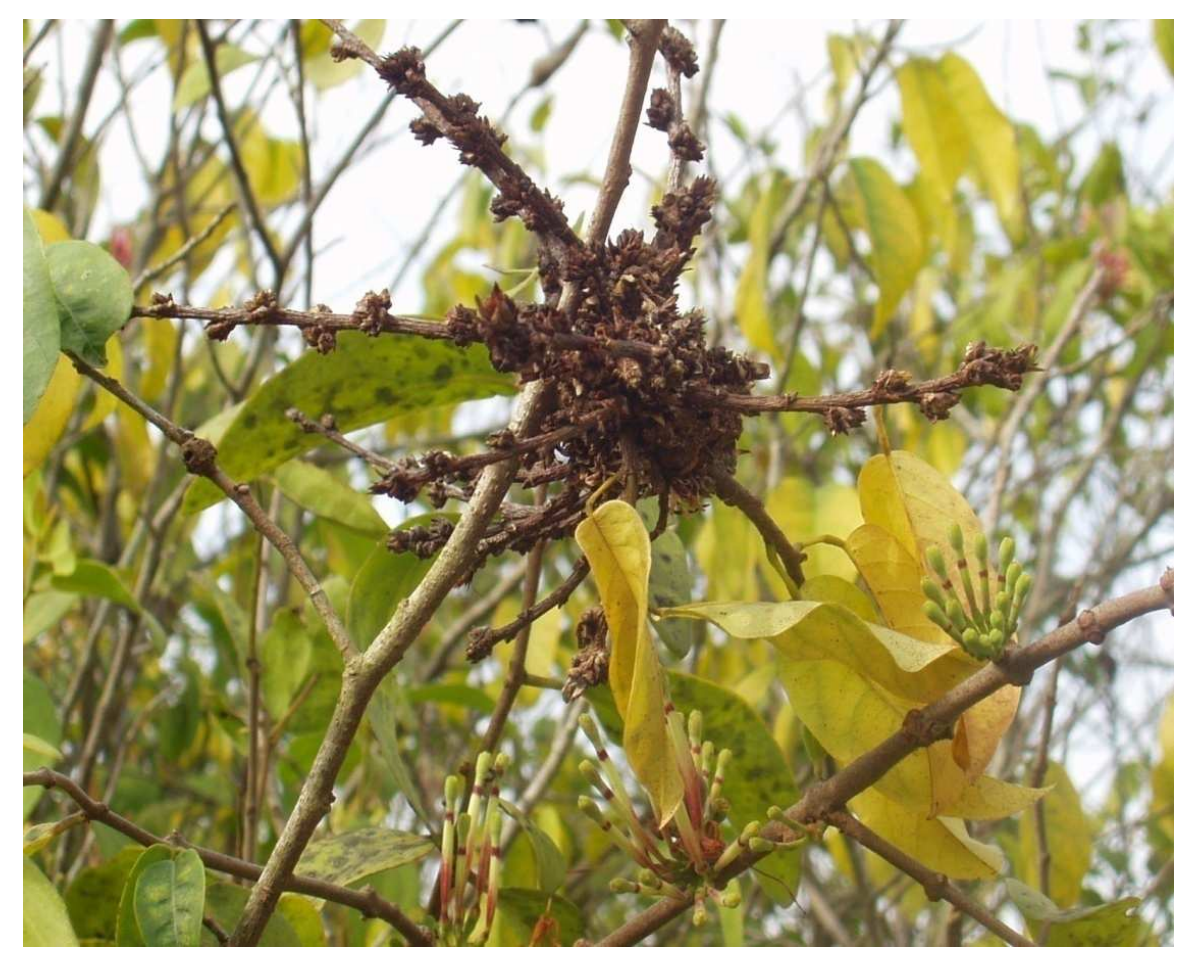

Figure 3: Rameaux feuillés et florifères de Tapinanthus belvisii (D C) Danser. 


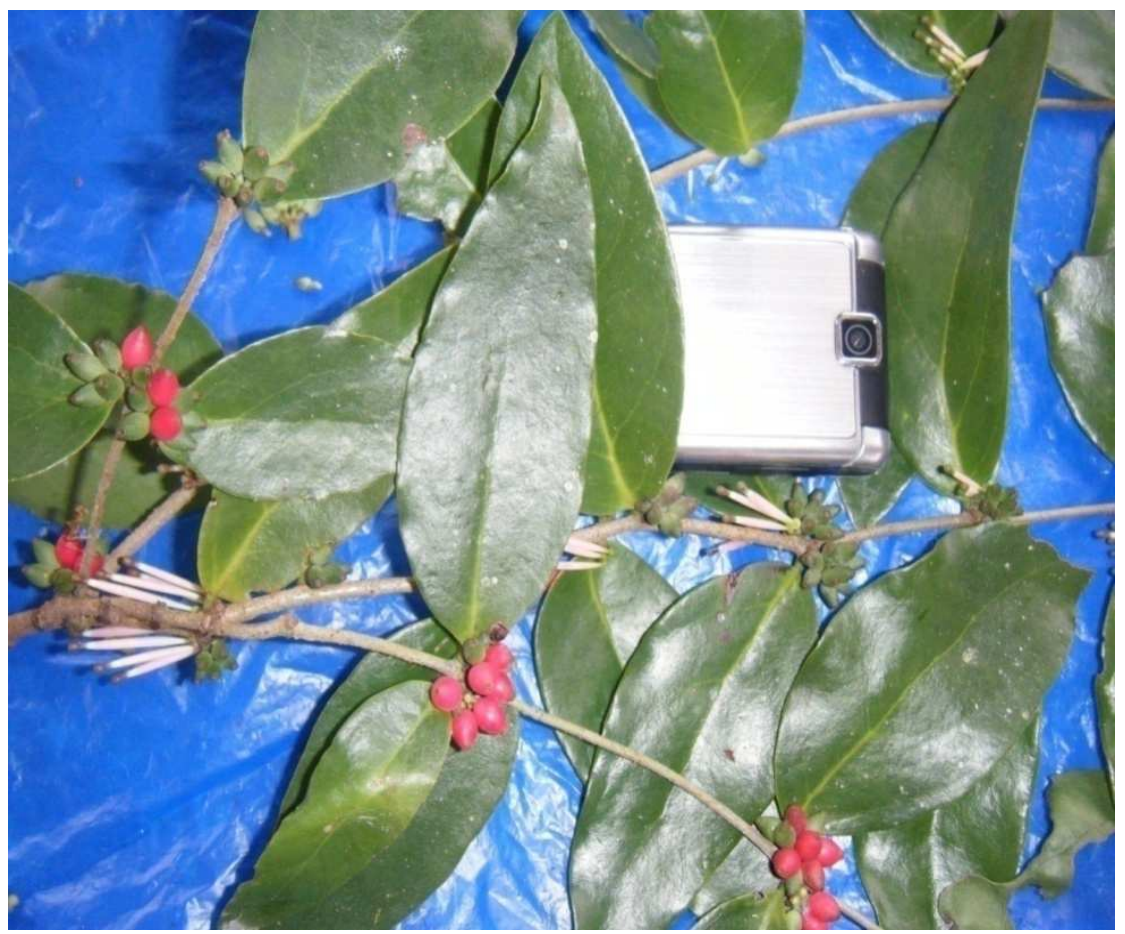

Figure 4: Rameaux florifères et fructifères de Tapinanthus sessilifolius var. glaber (P. Beauv.) Van Tiegh.

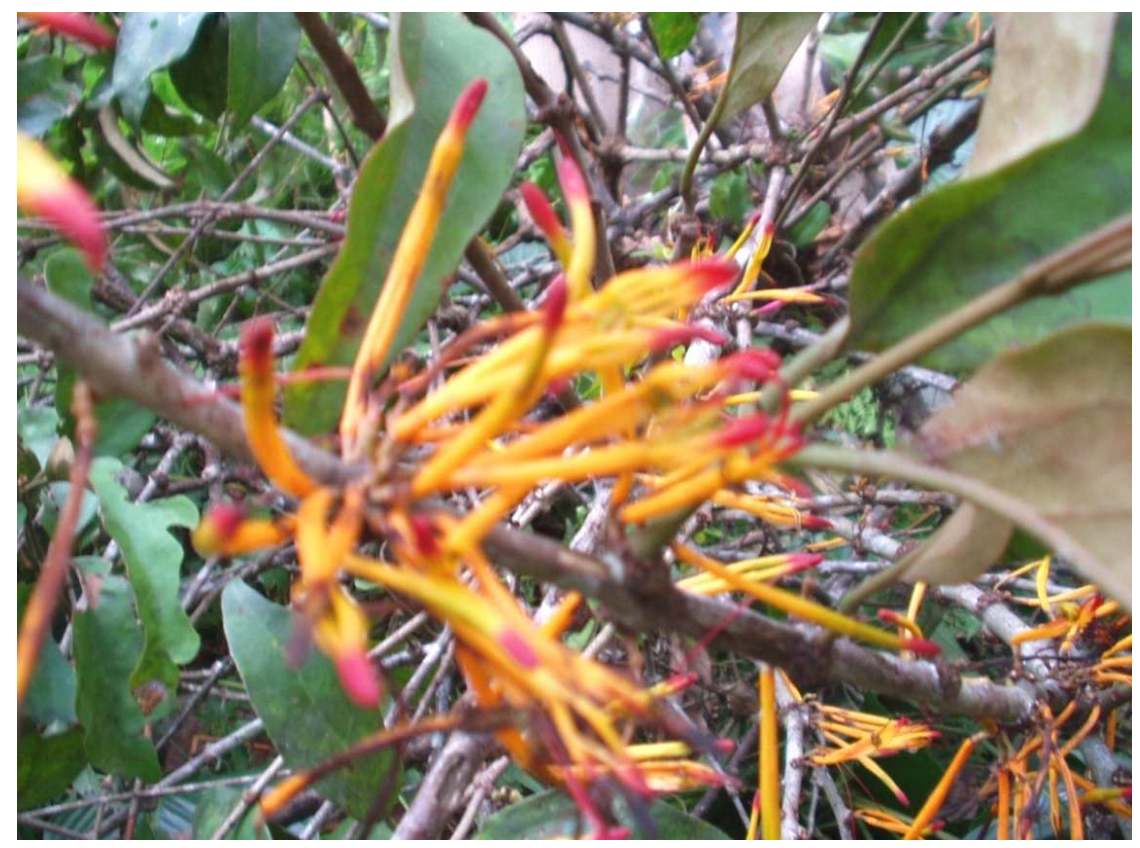

Figure 5: Rameaux florifères de Phragmanthera capitata (Spreng.) Ballé. 
D.E. A. AMON et al. / Int. J. Biol. Chem. Sci. 9(4): 1822-1834, 2015

Tableau 1: Flore ligneuse et état d'infestation des taxons hôtes selon l'inventaire.

\begin{tabular}{|c|c|c|c|c|c|c|}
\hline Genres \& espèces & Famille & Individus présents & Individus infestés & Total de touffes & Tx inf (p.c.) & Int inf \\
\hline Acacia mangium & Mimosaceae & 126 & 90 & 1764 & 71,42 & 19,6 \\
\hline Adansonia digitata & Bombacaceae & 6 & 2 & 7 & 33,33 & 3,5 \\
\hline Afzelia africana & Caesalpiniaceae & 137 & 27 & 112 & 19,7 & 4,14 \\
\hline Afzelia bipindensis & Caesalpiniaceae & 12 & 5 & 13 & 41,66 & 2,6 \\
\hline Albizia adianthifolia & Mimosaceae & 23 & 4 & 6 & 17,39 & 1,5 \\
\hline Albizia ferruginea & Mimosaceae & 7 & 3 & 6 & 42,85 & 2 \\
\hline Albizia zygia & Mimosaceae & 12 & 5 & 12 & 41,66 & 2,4 \\
\hline Alchornea cordifolia & Euphorbiaceae & 19 & 8 & 18 & 42,1 & 2,25 \\
\hline Alstonia boonei & Apocynaceae & 30 & 6 & 19 & 20 & 3,16 \\
\hline Amphimas pterocarpoides & Caesalpiniaceae & 18 & 6 & 9 & 33,33 & 1,5 \\
\hline Anacadium occidentale & Sapindaceae & 12 & 7 & 15 & 58,33 & 2,14 \\
\hline Annona muricata & Annonaceae & 88 & 9 & 27 & 10,22 & 3 \\
\hline Anthocleista djalonensis & Loganiaceae & 51 & 26 & 87 & 50,98 & 3,34 \\
\hline Anthocleista nobilis & Loganiaceae & 165 & 29 & 116 & 17,57 & 4 \\
\hline Antiaris toxicaria & Moraceae & 3 & 1 & 2 & 33,33 & 2 \\
\hline Azadirachta indica & Meliaceae & 12 & 2 & 15 & 16,66 & 7,5 \\
\hline Baphia bancoensis & Fabaceae & 4 & 2 & 5 & 50 & 2,5 \\
\hline Baphia nitida & Fabaceae & 480 & 105 & 195 & 21,87 & 1,85 \\
\hline Baphia pubescens & Fabaceae & 24 & 5 & 8 & 2083 & 1,6 \\
\hline Berlinia heudelotiana & Caesalpiniaceae & 7 & 2 & 8 & 28,57 & 4 \\
\hline Bixa orellena & Bixaceae & 4 & 1 & 2 & 35 & 2 \\
\hline Blighia sapida & Sapindaceae & 57 & 3 & 12 & 5,26 & 4 \\
\hline Blighia welwitschi & Sapindaceae & 4 & 2 & 4 & 50 & 2 \\
\hline Bombax buonopozense & Bombacaceae & 251 & 125 & 326 & 49,8 & 2,58 \\
\hline Bombax costatum & Bombacaceae & 5 & 3 & 6 & 60 & 2 \\
\hline Bridelia grandis & Euphorbiaceae & 53 & 4 & 6 & 7,54 & 1,5 \\
\hline Bridelia micrantha & Euphorbiaceae & 51 & 4 & 6 & 7,84 & 1,5 \\
\hline Caesalpinia bondouc & Caesalpiniaceae & 8 & 3 & 13 & 37,8 & 4,33 \\
\hline Callistemon speciosus & Myrtaceae & 14 & 4 & 9 & 28,57 & 2,25 \\
\hline
\end{tabular}


D.E. A. AMON et al. / Int. J. Biol. Chem. Sci. 9(4): 1822-1834, 2015

\begin{tabular}{|c|c|c|c|c|c|c|}
\hline Cassia alata & Caesalpiniaceae & 32 & 15 & 111 & 46,87 & 7,4 \\
\hline Cassia mimosoides & Caesalpiniaceae & 7 & 2 & 5 & 28,57 & 2,5 \\
\hline Cassia siamea & Caesalpiniaceae & 28 & 5 & 20 & 17,85 & 4 \\
\hline Cassia sieberiana & Caesalpiniaceae & 6 & 2 & 5 & 33,33 & 2,5 \\
\hline Cecropia peltata & Cecropiaceae & 226 & 192 & 1298 & 84,95 & 6,76 \\
\hline Celtis mildbraedii & Ulmaceae & 8 & 3 & 10 & 37,5 & 3,33 \\
\hline Chrysobalanus ellipticus & Chrysobalanaceae & 5 & 1 & 3 & 20 & 3 \\
\hline Citrus aurantium & Rutaceae & 46 & 11 & 71 & 23,91 & 6,45 \\
\hline Citrus grandis & Rutaceae & 14 & 5 & 13 & 35,71 & 2,6 \\
\hline Citrus limon & Rutaceae & 144 & 51 & 142 & 35,41 & 2,78 \\
\hline Citrus sinensis & Rutaceae & 410 & 112 & 741 & 27,31 & 6,61 \\
\hline Clappertonia minor & Tiliaceae & 12 & 3 & 4 & 25 & 1,33 \\
\hline Cleppertonia ficifolia & Tiliaceae & 21 & 4 & 15 & 19.04 & 3,75 \\
\hline Cnestis ferruginea & Connaraceae & 6 & 2 & 8 & 33,33 & 4 \\
\hline Coffea arabusta & Rutaceae & 352 & 37 & 73 & 10,51 & 1,97 \\
\hline Coffea canephora var. robusta & Rutaceae & 424 & 27 & 70 & 6,36 & 2,59 \\
\hline Cola caricifolia & Sterculiaceae & 2 & 1 & 2 & 50 & 2 \\
\hline Cola nitida & Sterculiaceae & 893 & 491 & 4754 & 54,98 & 9,68 \\
\hline Cordia platythyrsa & Boraginaceae & 5 & 2 & 4 & 40 & 2 \\
\hline Detarium microcarpum & Caesalpiniaceae & 12 & 2 & 5 & 16,66 & 2,5 \\
\hline Detarium senegalensis & Ebenaceae & 7 & 2 & 5 & 28,57 & 2,5 \\
\hline Diospyros crassifolia & Ebenaceae & 5 & 1 & 2 & 20 & 2 \\
\hline Diospyros mannii & Caesalpiniaceae & 23 & 7 & 21 & 30,43 & 3 \\
\hline Erythrina fusca & Fabaceae & 44 & 5 & 17 & 11,36 & 3,4 \\
\hline Eugenia jambos & Myrtaceae & 123 & 9 & 11 & 7,31 & 1,22 \\
\hline Eugenia malaccensis & Myrtaceae & 6 & 2 & 7 & 33,33 & 3,5 \\
\hline Eugenia miegeana & Myrtaceae & 8 & 3 & 14 & 37,5 & 4,66 \\
\hline Eugenia owariensis & Rubiaceae & 13 & 2 & 6 & 15,38 & 3 \\
\hline Ficus capensis & Moraceae & 7 & 2 & 5 & 28,57 & 2,5 \\
\hline Ficus exasperata & Moraceae & 280 & 25 & 121 & 8,92 & 4,84 \\
\hline
\end{tabular}


D.E. A. AMON et al. / Int. J. Biol. Chem. Sci. 9(4): 1822-1834, 2015

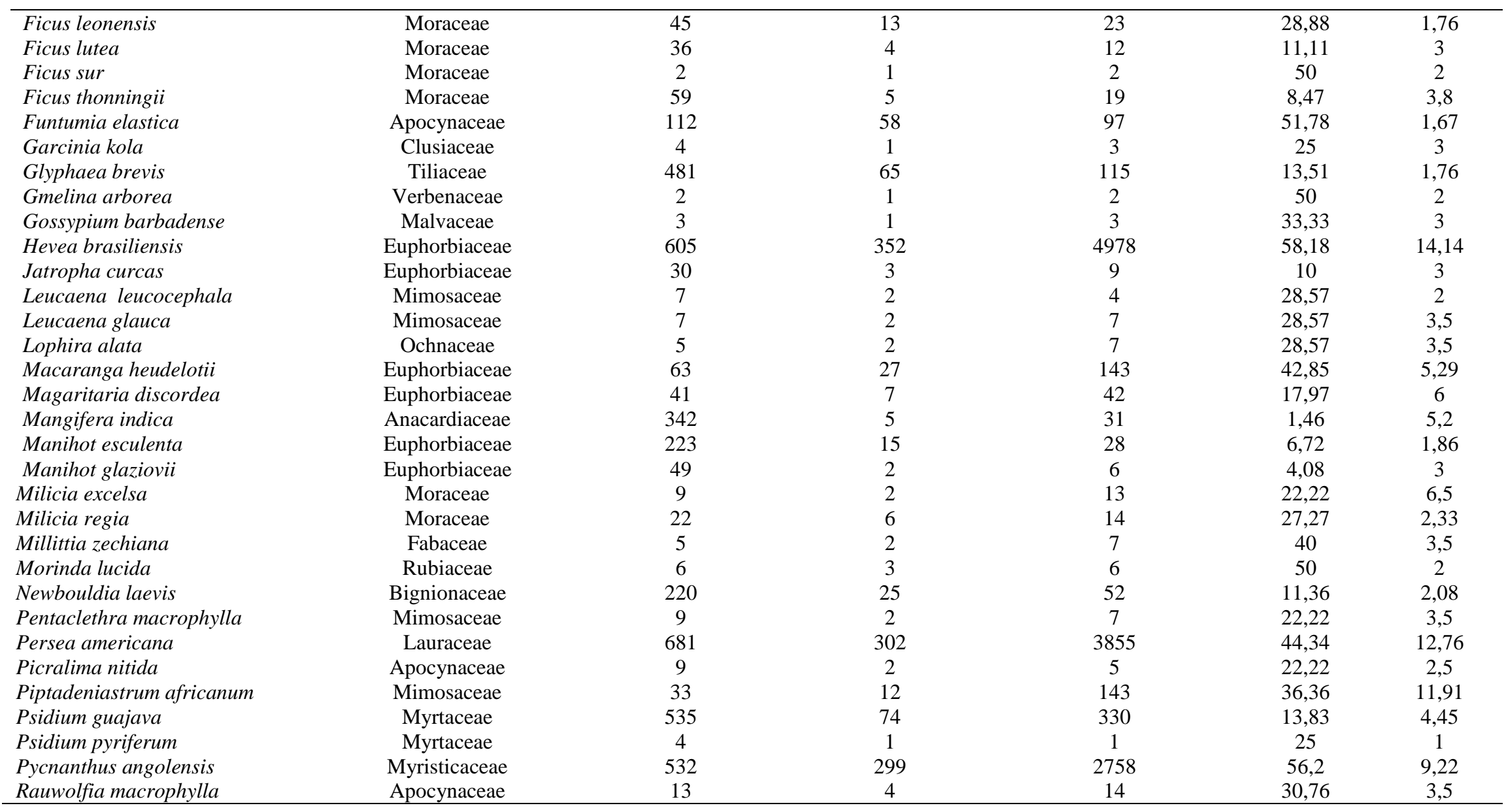


D.E. A. AMON et al. /Int. J. Biol. Chem. Sci. 9(4): 1822-1834, 2015

\begin{tabular}{|c|c|c|c|c|c|c|}
\hline Rauwolfia vomitoria & Apocynaceae & 438 & 197 & 959 & 44,97 & 4,86 \\
\hline Rhizophora racemosa & Rhizophoraceae & 3 & 1 & 13 & 33,33 & 13 \\
\hline Sabicea africana & Rubiaceae & 7 & 2 & 5 & 28,57 & 2,5 \\
\hline Solanum melongena & Solanaceae & 243 & 23 & 64 & 3,46 & 2,78 \\
\hline Spondias mombin & Anacardiaceae & 1244 & 939 & 6831 & 75,24 & 7,27 \\
\hline Spondias purpurea & Anacardiaceae & 5 & 2 & 6 & 40 & 3 \\
\hline Sterculia tragacantha & Sterculiaceae & 21 & 7 & 24 & 33,33 & 3,42 \\
\hline Tamarindus indica & Caesalpiniaceae & 9 & 2 & 5 & 22,22 & 2,5 \\
\hline Tarrieta utilis & Sterculiaceae & 5 & 2 & 9 & 40 & 4,5 \\
\hline Tectonia grandis & Verbenaceae & 124 & 21 & 279 & 16,93 & 13,28 \\
\hline Terminalia ivoriensis & Combretaceae & 9 & 3 & 11 & 33,33 & 3.66 \\
\hline Terminalia mantaly & Combretaceae & 4 & 1 & 5 & 25 & 5 \\
\hline Terminalia superba & Combretaceae & 6 & 2 & 7 & 33,33 & 3,5 \\
\hline Tetrapleura tetraptera & Mimosaceae & 23 & 6 & 28 & 26,08 & 4,66 \\
\hline Theobroma cacao & Sterculiaceae & 773 & 520 & 5854 & 67,27 & 11,25 \\
\hline Thevetia peruviana & Apocynaceae & 3 & 1 & 2 & 33,33 & 2 \\
\hline Treculia africana & Moraceae & 8 & 2 & 8 & 25 & 4 \\
\hline Trichilia djalonensis & Meliaceae & 5 & 2 & 7 & 40 & 3,5 \\
\hline Trichilia monadelpha & Meliaceae & 13 & 3 & 15 & 23,07 & 5 \\
\hline Xylopia aethiopica & Annonaceae & 27 & 12 & 34 & 44,44 & 2,83 \\
\hline Total & & 12086 & 4586 & 37383 & 37,94 & 8,15 \\
\hline Moyenne & & 105,1 & 39,87 & 325,06 & 37,94 & 8,15 \\
\hline
\end{tabular}

Tx inf -Taux d'infestation; Int inf - Intensités d'infestation. 
Tableau 2: Nombre de touffes par parasite inventorié sur les ligneux.

\begin{tabular}{ccccccccc}
\hline & & \multicolumn{7}{c}{ Espèces parasites } \\
\cline { 3 - 9 } & Total touffes & $\boldsymbol{T} \boldsymbol{b}$ & Tbel & $\boldsymbol{T s}$ & $\boldsymbol{P c v a}$ & $\boldsymbol{P c}$ & $\boldsymbol{G b \boldsymbol { r }}$ & $\boldsymbol{G \boldsymbol { d }}$ \\
\hline Total & 37383 & 14891 & 319 & 3930 & 6583 & 9231 & 2248 & 181 \\
Moyenne & 325,06 & 129,49 & 2,77 & 34,17 & 57,24 & 80,27 & 19,55 & 1,57 \\
& & 39,84 & 0,85 & 10,51 & 17,61 & 24,7 & 6,01 & 0,48 \\
\hline
\end{tabular}

Tb - Tapinanthus bangwensis; Tbel - Tapinanthus belvisii; Ts - Tapinanthus sessilifolius var. glaber; Pcva - Phragmanthera capitata var. alba; Pc - Phragmanthera; Gbr - Globimetula braunii; Gd - Globimetula dinklagei subsp. assiana.

\section{Infestation des ligneux par les plantes parasites}

Le taux d'infestation global des ligneux hôtes des Loranthaceae dans les agroécosystèmes est de 37,4 p.c. Ce taux est supérieur à celui obtenu par Soro et al. (2009) qui est de 29,05 p.c. dans l'essai Légumineuses/cacaoyers en zone forestière de la Côte d'Ivoire.

Les résultats relatifs à l'ampleur du fléau qu'est l'infestation des taxons hôtes montrent que les espèces ligneuses sont diversement attaquées par les parasites. La plupart des arbres fruitiers connus sont parasités par les Loranthaceae. Cola nitida, Persea americana, Psidium guajava et les agrumes (genre citrus) ont subi une forte infestation. Le parasitisme de ces fruitiers par les Loranthaceae est confirmé par Dibong et al. (2008).

Les résultats montrent aussi que Dolonix regia et Mangifera indica (manguier) résistant au parasitisme des Loranthaceae selon la littérature sont parasités dans la région d'étude. Ces résultats diffèrent de ceux de Dibong et al. (2008) qui les présentent comme des espèces résistantes aux parasites. Ces résultats, nous font penser qu'aujourd'hui, il n'y a certainement pas d'espèces résistantes aux hémiparasites, mais plutôt des espèces non encore découvertes parasitées par les Loranthaceae.

\section{Conclusion}

Les agroécosystèmes du Sud-Comoé, au Sud-Est de la Côte d'Ivoire abritent 7 espèces parasites. Le degré de parasitisme des ligneux par les Loranthaceae est fonction des hôtes selon l'inventaire. Tapinanthus bangwenis est la Loranthaceae ubiquiste la plus abondante des 7 espèces parasites inventoriées.

Les résultats ont révélé que la flore ligneuse des agroécosystèmes est attaquée à des degrés divers par les Loranthaceae. Dolonix regia et de Mangifera indica (manguier) considérées résistantes ont été trouvées parasitées par les Loranthaceae. Nous pouvons donc supposer qu'il n'existe pas d'espèces résistantes aux Loranthaceae, mais plutôt des espèces ligneuses non encore découvertes parasitées. Le parasitisme des Loranthaceae est donc une menace à considérer. En Côte d'Ivoire où l'économie repose sur l'agriculture, il est essentiel et impératif d'élaborer des programmes de recherche de lutte ciblée pour les essences fruitières, source de revenu pour les paysans.

\section{Conflits d'intérêts}

Les auteurs déclarent qu'il n'existe aucun conflit d'intérêt dans cet article.

\section{Contributions des auteurs}

DEAA était l'investigateur principal. Il a effectué les travaux de terrain et les tests de laboratoire. DS et DT ont conçu le projet et supervisé le travail. Tous ces auteurs ont participé à la rédaction du manuscrit.

\section{REFERENCES}

Aké-Assi L. 2001. Flore de la Côte d'Ivoire 1, Catalogue, Systématique, biogéographie et Ecologie. Conservatoire et Jardin Botanique: Genève, Switzerland; 396p

Amon ADE. 2006. Les plantes vasculaires parasites de la famille des Loranthaceae rencontrées dans le Département de Grand-Bassam, au Sud de la Côte d'Ivoire. Mémoire de DEA de Botanique, Université de Cocody, UFR Biosciences Abidjan, Côte d'Ivoire, $57 \mathrm{p}$.

Amon ADE. 2014. Les Loranthaceae (guis), hémiparasites vasculaires des arbres et des arbustes des agroécosystèmes de la région du Sud-Comoé, en zone de forêt 
dense sempervirente de la Côte d'Ivoire. Mémoire de Thèse de l'Université Félix Houphouët-Boigny, option Agroforesterie, 213p.

Anader. 2003a. Monographie du département d'Aboisso. Anader, zone Aboisso, Côte d'Ivoire, 56p.

Anader. 2003b. Monographie du département d'Adiaké. Anader, zone Adiaké, Côte d'Ivoire, 53p.

Boussim IJ. 2002. Les Phanérogames parasites $\mathrm{du}$ Burkina Faso : inventaire, taxonomie, écologie, et quelques aspects de leur biologie: Cas particulier des Loranthaceae parasites du karité. Thèse de Doctorat d'État ès Sciences Naturelles, FAST, Université de Ouagadougou, Burkina Faso, 285p.

Boussim IJ, Yonli D, Medah NM, Guinko S, Sallé G. 2012. Prospects for an integrated of Loranthaceae species parasitizing Vitellaria paradoxa C.F. Gaertn in Burkina Faso. International Journal of Biological and Chemical Sciences, 6(1): 355 - 364.

Dibong S, Engone Obiang NL, Din N, Priso RJ, Taffouo VD, Fankem H, Amougou A. 2008. Parasitism of host trees by the Loranthaceae in the religion of Douala (Cameroun) in the region of Douala (Cameroun). African Journal of Environmental Science et Technology, 2(12): 412-421.

Dibong SD, Engone ONL, Din N, Priso RJ, Taffouo VD, Fankem H, Salle G, Amougou A. 2009. Niveau d'infestation des arbres fruitiers des groupements végétaux par Phragmanthera capitata (Sprengel) S.
Balle (Loranthaceae) dans la région du littorale du Cameroun. Int. J. Biol. Chem. Sci., 3(2): 347-354.

Hall, Swaine. 1981. Distribution and ecology of vascular plants in a rain forest vegetation in Ghana. Geobotany; 383p.

Koua A. 2007. Situation de la production de café en Côte d'Ivoire: cas du département d'Aboisso. BNETD, 90p.

Salle G. 2004. Les plantes parasites, 14 p. http://www.futurasciences.com/magazi nes/botanique plantes parasites [Consulté le 5/07/2014].

Soro D, Ouattara D, PK Da, D Traoré. 2004. Efficacité de l'émondage contre les Loranthaceae ou guis du karité : cas du parc naturel à karité de Tengrela dans le Nord de la Côte d'Ivoire. Annales de Botanique de l'Afrique de l'Ouest, 16(3): 21-28.

Soro K. 2010. Les Loranthaceae (guis) des agroécosystèmes dans l'Ouest de la Côte d'Ivoire: flore, parasitisme et usages dans les Départements de Oumé, de Gagnoa et de Soubré. Mémoire de Thèse de l'Université de Cocody-Abidjan, option Agroforesterie, 183p.

Soro K, Gnahoua GM, Traore D. 2009. Parasitisme des Loranthaceae dans les plantations de Légumineuses arborescentes en zone forestière de la Côte d'Ivoire. Agronomie Africaine, 21(1): 59-69. 